\title{
Expression of matrix metalloproteinases and endogenous inhibitors within ascending aortic aneurysms of patients with bicuspid or tricuspid aortic valves
}

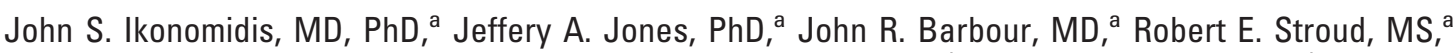
Leslie L. Clark, MS, ${ }^{\text {a }}$ Brooke S. Kaplan, BS, ${ }^{\text {a }}$ Ahmed Zeeshan, MD, Joseph E. Bavaria, MD, ${ }^{\text {b }}$ Joseph H. Gorman III, MD, Francis G. Spinale, MD, PhD, and Robert C. Gorman, MD ${ }^{b}$

From the Department of Cardiothoracic Surgical Research, ${ }^{\text {a }}$ Division of Cardiothoracic Surgery, Medical University of South Carolina, Ralph H. Johnson Veterans Affairs Medical Center, Charleston, South Carolina; and Division of Cardiothoracic Surgery, ${ }^{\mathrm{b}}$ University of Pennsylvania, Philadelphia, Penn.

This work was supported by NIH/NHLBI R01 Grants HL075488-01, HL 059165-07 and Research Institute of the Department of Veterans Affairs Award.

Read at the Eighty-sixth Annual Meeting of The American Association for Thoracic Surgery, Philadelphia, Pa, April 29-May 3, 2006.

Received for publication April 27, 2006; revisions received Sept 27, 2006; accepted for publication Oct 9, 2006.

Address for reprints: John S. Ikonomidis, $\mathrm{MD}, \mathrm{PhD}$, Associate Professor of Surgery, Division of Cardiothoracic Surgery, Medical University of South Carolina, Suite 409 CSB, 96 Jonathan Lucas Street, Charleston, SC 29425 (E-mail: ikonomij@musc.edu).

J Thorac Cardiovasc Surg 2007;133:1028-36 $0022-5223 / \$ 32.00$

Copyright $\odot 2007$ by The American Association for Thoracic Surgery

doi:10.1016/j.jtcvs.2006.10.083
Objective: The mechanisms contributing to ascending thoracic aortic aneurysms associated with bicuspid aortic valves may differ from ascending thoracic aortic aneurysms with tricuspid aortic valves. Matrix metalloproteinases and their endogenous inhibitors have been causally linked to ascending thoracic aortic aneurysm formation. This study tested the hypothesis that specific and different matrix metalloproteinase and tissue inhibitors of metalloproteinase profiles would be observed in ascending thoracic aortic aneurysm samples from patients with bicuspid aortic valves versus tricuspid aortic valves.

Methods: Ascending thoracic aortic aneurysm samples taken from patients with bicuspid aortic valve $(n=53)$ and patients with tricuspid aortic valve $(n=46)$ were assessed for representative subtypes of all matrix metalloproteinase classes and all 4 known tissue inhibitors of metalloproteinases. Levels were compared [optical density units, median (interquartile range)] both to reference control ascending aortic samples $(\mathrm{n}=26)$ and within each valve group by aneurysm diameter $(\leq 3.9 \mathrm{~cm}, 4.0-5.9 \mathrm{~cm}$ and $\geq 6.0 \mathrm{~cm})$.

Results: Different and specific matrix metalloproteinase and tissue inhibitors of metalloproteinase profiles were observed in the ascending thoracic aortic aneurysm groups. In bicuspid aortic valves, matrix metalloproteinase- 2 increased by $34 \%$ when compared with either tricuspid aortic valves or control $(P<.05)$, and matrix metalloproteinase-14 decreased by 59\% compared with tricuspid aortic valves $(P<.05)$. In tricuspid aortic valve samples, tissue inhibitors of metalloproteinase- 2 decreased by $35 \%$ when compared with either tricuspid aortic valves or control $(P<.05)$, and matrix metalloproteinase-13 increased by $140 \%$ in the 4.0- to $5.9-\mathrm{cm}$ diameter range $(P<.05)$.

Conclusions: A unique matrix metalloproteinase and tissue inhibitor of metalloproteinase portfolio was observed in ascending thoracic aortic aneurysms from patients with bicuspid aortic valve compared with patients with tricuspid aortic valve. These differences, suggesting disparate mechanisms of extracellular matrix remodeling, may provide unique biochemical targets for ascending thoracic aortic aneurysm prognostication and treatment in these 2 groups of patients.

$\mathrm{H}$ istorically, research regarding the underlying basis for ascending thoracic aortic aneurysm (ATAA) formation has been limited to Marfan's syndrome and other connective tissue disorders. More recently, interest has shifted to the more common conditions associated with ATAAs. ${ }^{1-4}$ These include nonspecific medial degenerative disease associated with tricuspid aortic valves (TAVs) and ATAAs associated with congenital bicuspid aortic valves (BAVs). ${ }^{1-4}$ Recent work has shown that differences between these 2 ATAA populations exist at both the 


\section{Abbreviations and Acronyms}

ATAA $=$ ascending thoracic aortic aneurysm

$\mathrm{BAV}=$ bicuspid aortic valve

MMP = matrix metalloproteinase

TAV $=$ tricuspid aortic valve

TIMP $=$ tissue inhibitors of metalloproteinase

$\mathrm{TNF}=$ tumor necrosis factor

molecular and histologic level but that these differences remain incompletely characterized. ${ }^{1-4}$ A central theme with regard to ATAA formation is disturbances in proteolytic activity within the aneurysm wall..$^{5-7}$ The matrix metalloproteinases (MMPs) constitute a large family of enzymes that process or degrade the extracellular matrix. The MMPs are grouped into classes based on their primary substrate specificities. An association has been established between increases in MMPs and development of aortic aneurysms. ${ }^{5,6}$ Primary endogenous control of MMP activity is maintained through 4 tissue inhibitors of metalloproteinases (TIMPs). ${ }^{7}$ In aortic aneurysms, imbalances in the ambient MMP and TIMP profiles within the aortic wall are observed that favor net proteolysis. ${ }^{6}$

The possible contribution of changes in MMP and TIMP profiles within the aortic wall of ATAAs from patients with BAV has been incompletely quantified. Further, whether and to what degree these profiles differ from ATAAs from patients with TAV is unknown. Accordingly, the present study tested the hypothesis that specific differences exist with respect to the MMP and TIMP cassettes expressed in ATAAs in patients with BAV versus patients with TAV.

\section{Materials and Methods \\ Study Population}

The study population consisted of ascending aortic specimens from 53 patients with BAV and 46 patients with TAV taken at the time of aneurysm resection or aortic valve replacement. A reference control group $(n=26)$ was made up of ascending aortic specimens taken from 1 patient at coronary bypass, 14 heart transplant recipients, and 11 heart or lung donors. No patient had an aortic dissection, connective tissue disorder, or inflammatory aortic disease. For ascending aortic diameters less than $4.0 \mathrm{~cm}$, aortic resection was not performed but a biopsy was taken from the aortotomy site during aortic valve replacement. There were no gender differences between the groups (control: $76 \%$ men; BAV: $75 \%$ men; TAV: $59 \%$ men, $P=.14 \chi^{2}$ ). The ages within the groups were as follows: median (interquartile range): control, 52 (18) years; BAV, 60 (14) years; TAV, $72(15)$ years $(P<.05$ BAV/TAV from control, TAV from BAV). The aortic diameters [median (interquartile range)] were not different between ATAA groups but different from control: control, $2.8(0.3) \mathrm{cm}$; BAV, 5.1 (0.7) cm; TAV, $5.5(1.6) \mathrm{cm}(P<.05 \mathrm{BAV} / \mathrm{TAV}$ from control).

The study was approved by the Institutional Review Boards of both The Medical University of South Carolina and The University of Pennsylvania. Informed consent was obtained from all patients.

\section{Immunoblotting and Zymography}

Aortic sample preparations. The resected specimens were placed in tubes and snap-frozen in a dry ice/methanol slurry prior to storage at $-80^{\circ} \mathrm{C}$. For analysis, the samples were homogenized in a cold acidic extraction buffer solution to prevent proteolytic activation during the extraction process. ${ }^{8}$ The homogenate was then centrifuged $\left(4^{\circ} \mathrm{C}, 10\right.$ minutes, $\left.1200 \mathrm{~g}\right)$, the supernatant concentrated (Centriplus; Millipore, Bedford, Mass), and the final protein concentration of the aortic extracts was determined (BCA Protein Assay; Pierce, Rockford, Ill).

Zymography. Relative aortic MMP gelatinase activity was examined by substrate-specific zymographic analysis as described previously. ${ }^{9}$ The aortic extracts $(10 \mu \mathrm{g}$ total protein) were loaded onto electrophoretic gels (sodium dodecylsulfate-polyacrylimide gel electrophoresis) containing $1 \mathrm{mg} / \mathrm{mL}$ of gelatin followed by washing and incubation in an MMP substrate buffer for 12 hours at $37^{\circ} \mathrm{C}$. After incubation, the gels were stained using $0.1 \%$ Coomassie blue and destained in water. An MMP-2/-9 recombinant standard (CC073, Chemicon, Temecula, Calif) was added to each gel to serve as a positive control.

Immunoblotting. The relative abundances of MMPs/TIMPs were examined by using quantitative immunoblotting techniques. ${ }^{8,9}$ Briefly, $10 \mu \mathrm{g}$ of aortic extract was loaded onto a $4 \%$ to $12 \%$ Bis-Tris gradient gel (Invitrogen Corp, Carlsbad, Calif) and subjected to electrophoresis. The fractionated proteins were transferred to nitrocellulose membranes $(0.45 \mu \mathrm{m}$, Bio-Rad, Hercules, Calif) and subsequently blocked with 5\% nonfat dry milk in phosphate-buffered saline solution (PBS) for 1 hour at room temperature. The membranes were incubated in diluted antiserum $(0.4 \mu \mathrm{g} / \mathrm{mL}$ in $5 \%$ nonfat dry milk/PBS $)$ containing specific antibodies representative of all nongelatinase MMP classes (collagenases, elastases, stromelysins, matrilysins, and the membrane-type MMPs) and all known TIMPs (see Appendix). Following incubation with the primary antibody, the membranes were extensively washed $(3 \times 10$ minutes, $25 \mathrm{~mL}$ PBS $)$, and a secondary peroxidase-conjugated antibody (species-dependent on primary antibody used) was applied (1:5000, 5\% nonfat dry milk/ PBS) and incubated for 1 hour at room temperature. Following washing $(4 \times 15$ minutes, $25 \mathrm{~mL}$ PBS), immunoreactive signals were detected by briefly incubating the membranes with a chemiluminescent substrate (Western Lightning Chemiluminescence Reagent Plus, Perkin Elmer, Piscataway, NJ) and exposing the blot to film (Hyperfilm, GE Healthcare, Piscataway, NJ).

\section{Data Analysis}

The zymograms and immunoblot films were digitized and quantitative image analysis (Gel Pro Analyzer; Media Cybernetics, Silver Spring, Md) was performed.

All statistical procedures were performed using the Stata statistical software package (Stata Corporation, College Station, Tex). The analysis was conducted in 2 parts. For the first analysis, all samples in the BAV and TAV groups with an ascending size less than $4.0 \mathrm{~cm}$ were excluded. The MMP and TIMP data distributions for the reference controls, BAV group, and TAV group were tested for normality using the Shapiro-Wilk test. The results indicated nonparametricity, and hence a two-sample Mann-Whitney rank sum test was performed to test comparisons between groups. 
TABLE 1. MMP and TIMP immunoblotting results (median [interquartile range])

\begin{tabular}{|c|c|c|c|c|}
\hline & & Reference & & \\
\hline & Analyte & $\begin{array}{l}\text { Controls } \\
(n=26)\end{array}$ & $\begin{array}{c}\text { BAV } \\
(n=50)\end{array}$ & $\begin{array}{c}\text { TAV } \\
(n=37)\end{array}$ \\
\hline Collagenases & MMP-1 & 91 (117) & $69(49)$ & $52(49)$ \\
\hline & MMP-8 & $54(78)$ & $92(90)$ & $90(86)$ \\
\hline & MMP-13 & 71 (144) & 71 (109) & $91(300)$ \\
\hline Gelatinases & MMP-2 & $93(86)$ & $140(77)^{*}$ & $92(41) \dagger$ \\
\hline & MMP-9 & $56(99)$ & $52(65)$ & $61(100)$ \\
\hline Lysins & MMP-3 & 67 (102) & $41(72)$ & $43(50)^{*}$ \\
\hline & MMP-7 & 72 (105) & 78 (116) & 97 (227) \\
\hline Membrane type & MMP-14 & $59(66)$ & $38(61)^{*}$ & $92(78) \dagger$ \\
\hline & MMP-15 & $88(57)$ & $97(58)$ & $87(66)$ \\
\hline Elastase & MMP-12 & $80(75)$ & $80(74)$ & $67(68)$ \\
\hline Inhibitors & TIMP-1 & $67(101)$ & 59 (150) & $98(133)$ \\
\hline & TIMP-2 & $80(42)$ & $79(49)$ & $52(37)^{*}+\dagger$ \\
\hline & TIMP-3 & $63(130)$ & $61(96)$ & $51(126)$ \\
\hline & TIMP-4 & $80(56)$ & $76(54)$ & 104 (108) \\
\hline
\end{tabular}

$M M P$, Matrix metalloproteinase; TIMP, tissue inhibitors of metalloproteinase; $B A V$, bicuspid aortic valve; $T A V$, tricuspid aortic valve. $* P<.05$ vs reference control. $† P<.05$ vs BAV.

For the second portion of the analysis, the raw data was categorized by ascending aortic size: $\leq 3.9 \mathrm{~cm}, 4.0-5.9 \mathrm{~cm}$, and $\geq 6.0 \mathrm{~cm}$. The $\leq 3.9-\mathrm{cm}$ group was composed of all aortic samples with diameters $\leq 3.9 \mathrm{~cm}$ across the 3 groups (26 reference controls, 3 BAVs, and 9 TAVs; $n=38$ ). Spearman's rank correlation coefficient testing was used to determine correlation between ascending aortic size as categorized and MMPs/TIMPs. Comparisons within the BAV and TAV groups were performed using a two-sample Mann-Whitney rank sum test.

Regression analyses were also performed testing for the effects of age, hypertension, aortic valve pathology (stenosis versus regurgitation), and aortic diameter indexed to body surface area. ${ }^{10}$

Data were calculated as a percentage of the reference control group set to a mean of $100 \%$ and expressed as the median (interquartile range). As such, in the succeeding figures, the control values will deviate from 100 due to expression of the median, not the mean, value.

\section{Results}

Overall Analysis

The aortic samples were assayed for MMP species representing all of the known classes and are given in Table 1, with representative immunoblots and zymograms given in Figure 1. Within the gelatinase (MMP-2, MMP-9) class, MMP-2 was increased in the BAV group relative to the TAV and reference control groups, with no significant differences observed with MMP-9. In the membrane-type MMP class (MMP-14, MMP-15), MMP-14 was decreased in the BAV group relative the TAV and reference control values. In the stromelysin/matrilysin (MMP-3, MMP-7) class, MMP-3 was decreased in the TAV group relative to

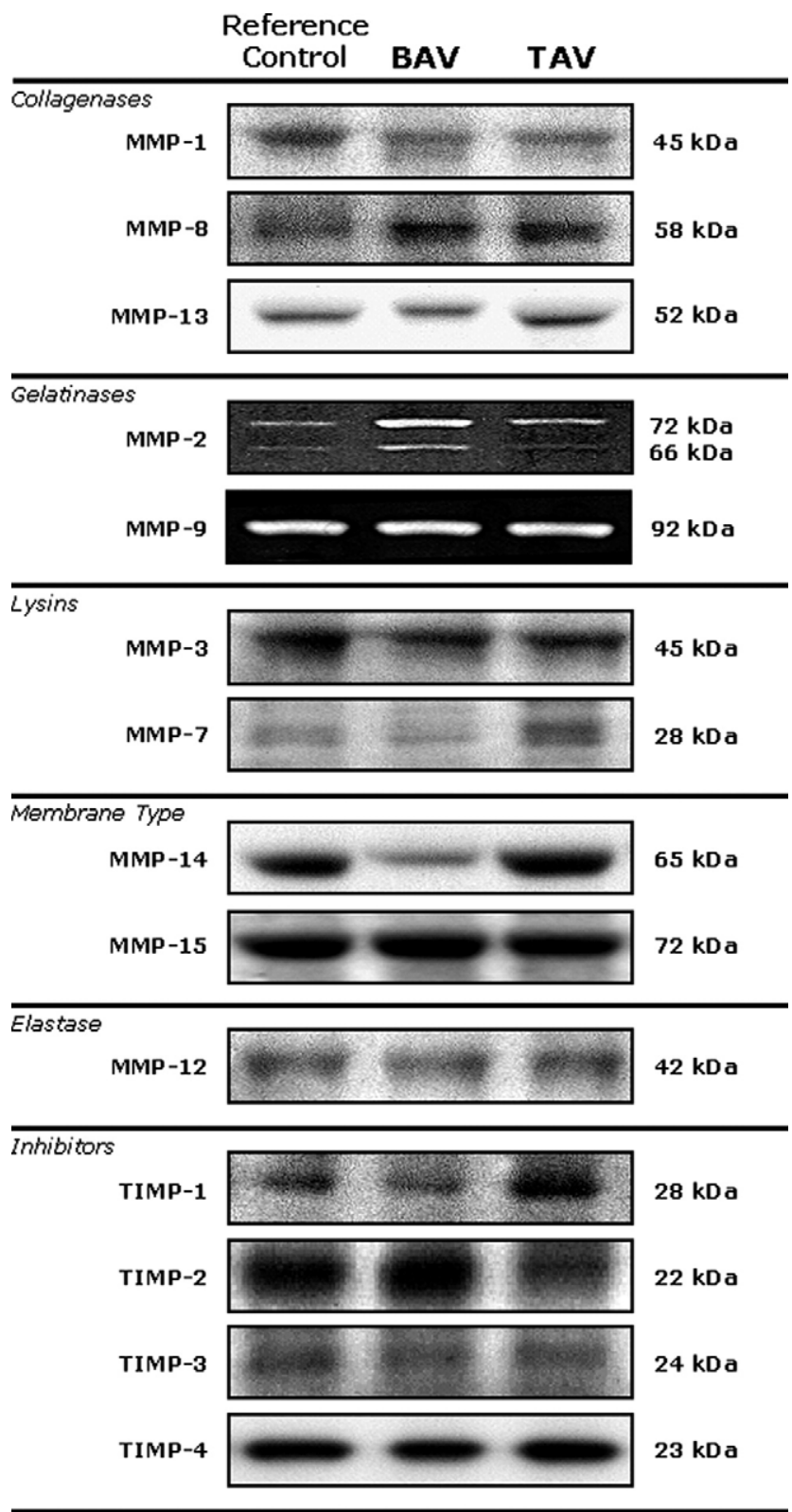

Figure 1. Representative immunoblots and zymographic analysis of the MMPs and TIMPs in reference control, BAV, and TAV ascending aortic samples. MMP, Matrix metalloproteinase; TIMP, tissue inhibitors of metalloproteinase; BAV, bicuspid aortic valve; TAV, tricuspid aortic valve.

control values. No differences were observed in the collagenase (MMP-1, MMP-8, MMP-13) or elastase (MMP-12) classes.

In this study the aortic samples were surveyed for all TIMP subtypes. As shown in Table 1, TIMP-2 was decreased in the TAV group relative to BAV group and control values. 


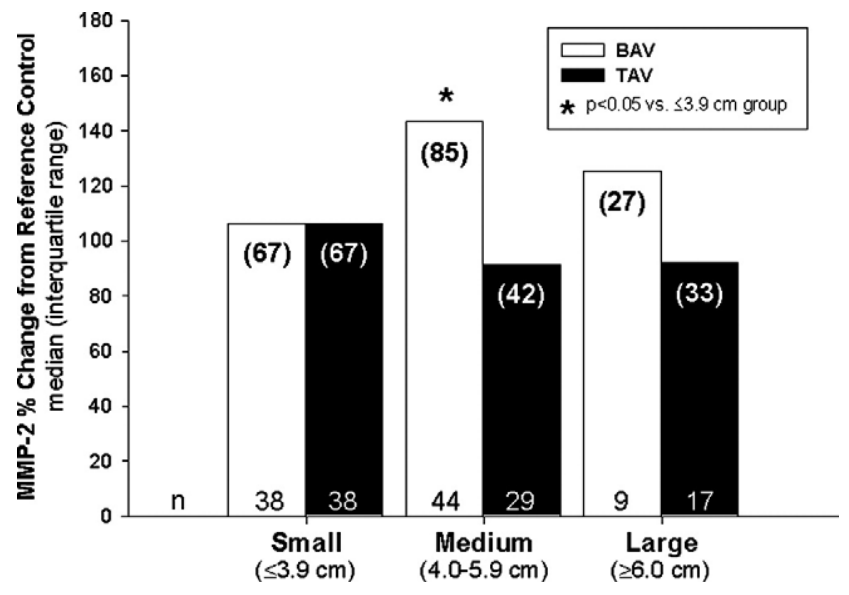

Figure 2. Comparison of MMP-2 levels for BAV and TAV aneurysms by aneurysm size. MMP-2 was increased in the medium BAV aneurysm group relative to the small BAV aneurysm group. $M M P$, Matrix metalloproteinase; BAV, bicuspid aortic valve; TAV, tricuspid aortic valve.

Size Analysis: BAV

In the BAV group, Spearman's testing revealed weak but significant correlations between MMP-2 (Figure 2, rho value $0.243, P=.02$ ) and MMP-14 (Figure 3, rho value $-0.313, P=.003)$ and aneurysm size.

Examination by categorized size (Table 2) showed increased MMP-2 and decreased MMP-3 and MMP-14 in the 4.0- to 5.9-cm diameter size range compared with the $\leq 3.9$-cm group. TIMP- 1 was decreased in the $\geq 6.0$-cm size range relative to the $\leq 3.9-\mathrm{cm}$ group.

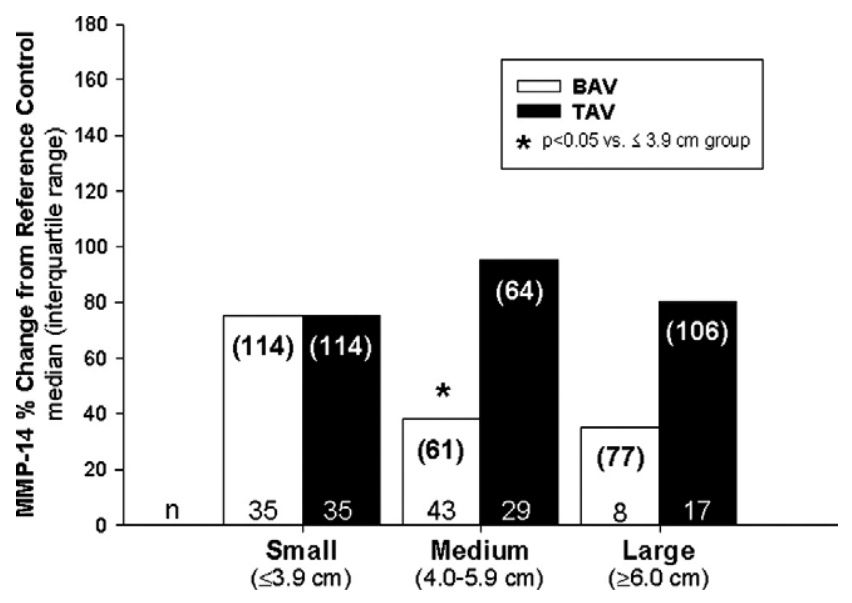

Figure 3. Comparison of MMP-14 levels for BAV and TAV aneurysms by aneurysm size. In the BAV cohort, MMP-14 decreased in the medium aneurysm group compared with the small aneurysm group. MMP, Matrix metalloproteinase; BAV, bicuspid aortic valve; TAV, tricuspid aortic valve.
TABLE 2. MMP and TIMP in BAV by ascending size (median [interquartile range])

\begin{tabular}{|c|c|c|c|c|}
\hline BAV & Analyte & $\begin{array}{l}\leq 3.9 \mathrm{~cm} \\
(\mathrm{n}=38)\end{array}$ & $\begin{array}{c}4.0-5.9 \mathrm{~cm} \\
(\mathrm{n}=44)\end{array}$ & $\begin{array}{l}\geq 6.0 \mathrm{~cm} \\
(\mathrm{n}=9)\end{array}$ \\
\hline \multirow[t]{3}{*}{ Collagenases } & MMP-1 & 83 (103) & $70(57)$ & $65(38)$ \\
\hline & MMP-8 & $69(77)$ & $96(90)$ & $76(97)$ \\
\hline & MMP-13 & $50(133)$ & $68(116)$ & $94(95)$ \\
\hline \multirow[t]{2}{*}{ Gelatinases } & MMP-2 & $106(67)$ & $143(85)^{*}$ & $125(27)$ \\
\hline & MMP-9 & $45(89)$ & $52(94)$ & $53(42)$ \\
\hline \multirow[t]{2}{*}{ Lysins } & MMP-3 & $67(81)$ & $37(60)^{*}$ & $91(76)$ \\
\hline & MMP-7 & $85(80)$ & $82(123)$ & $57(78)$ \\
\hline \multirow[t]{2}{*}{ Membrane type } & MMP-14 & 75 (114) & $38(61)^{*}$ & $35(77)$ \\
\hline & MMP-15 & $85(34)$ & $96(69)$ & $123(18)$ \\
\hline Elastase & MMP-12 & $74(67)$ & $79(78)$ & 101 (195) \\
\hline \multirow[t]{4}{*}{ Inhibitors } & TIMP-1 & 72 (99) & $68(151)$ & $36(25)^{*}$ \\
\hline & TIMP-2 & $80(41)$ & $79(50)$ & $83(39)$ \\
\hline & TIMP-3 & $48(77)$ & $58(65)$ & 102 (158) \\
\hline & TIMP-4 & $79(67)$ & $75(52)$ & $105(61)$ \\
\hline
\end{tabular}

$M M P$, Matrix metalloproteinase; TIMP, tissue inhibitors of metalloproteinase; $B A V$, bicuspid aortic valve. $* P 0.05$ vs $<3.9-\mathrm{cm}$ group.

\section{Size Analysis: TAV}

In the TAV group, Spearman's testing revealed significant correlations between MMP-7 (Figure 4, rho value 0.229, $P=.04$ ) and TIMP-2 (Figure 5, rho value $-0.288, P=$ .009) and aneurysm size.

Examination by categorized size (Table 3 ) showed increased MMP-13 (Figure 6) in the 4.0- to 5.9-cm diameter size range compared with the $\leq 3.9-\mathrm{cm}$ group followed by a reduction in the $\geq 6.0-\mathrm{cm}$ size range. In addition, decreased MMP-1, MMP-3, and TIMP-2 were demonstrated in the $4.0-$ to $5.9-\mathrm{cm}$ diameter size range compared with the

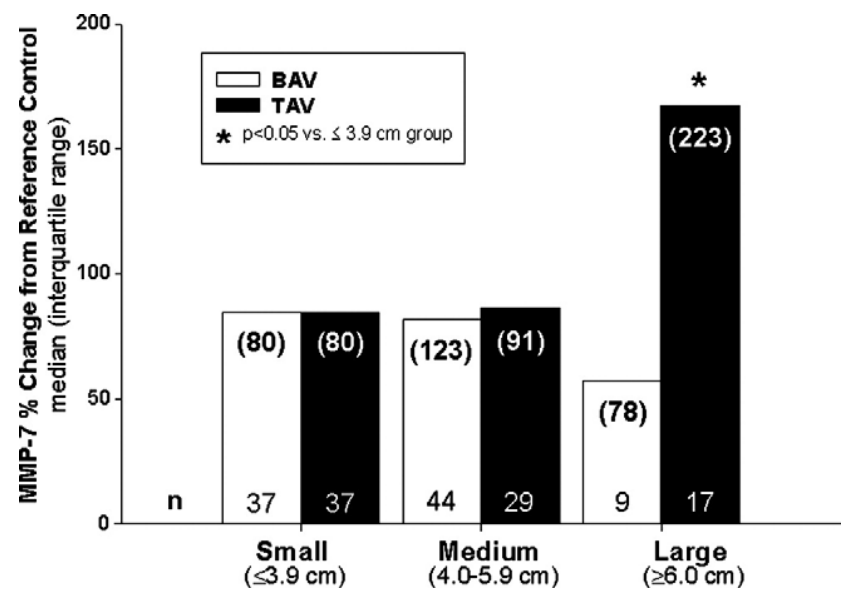

Figure 4. Comparison of MMP-7 levels for BAV and TAV aneurysms by aneurysm size. In the TAV cohort, MMP-7 was increased in the large aneurysm group compared with the small aneurysm group. MMP, Matrix metalloproteinase; BAV, bicuspid aortic valve; TAV, tricuspid aortic valve. 


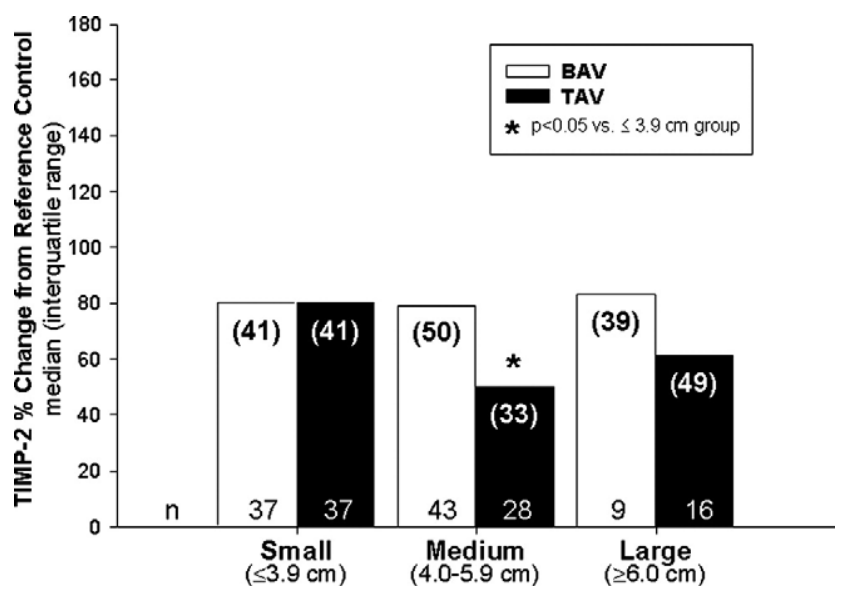

Figure 5. Comparison of TIMP-2 levels for BAV and TAV aneurysms by aneurysm size. In the TAV cohort, TIMP-2 decreased in the medium aneurysm group compared with the small aneurysm group. TIMP, tissue inhibitors of metalloproteinase; $B A V$, bicuspid aortic valve; TAV, tricuspid aortic valve.

$\leq 3.9$-cm group. MMP-7 was seen to be increased (Figure 4) in the in the $\geq 6.0-\mathrm{cm}$ size range compared with the $\leq 3.9-\mathrm{cm}$ group.

\section{Regression Analyses}

Regression analysis examining the affect of age on MMP levels showed a positive effect on MMP-13 in the BAV group $(P=.021)$. A positive correlation was observed between MMP-1 and presence of hypertension $(P=.02)$

TABLE 3. MMP and TIMP in TAV by ascending size (median [interquartile range])

\begin{tabular}{|c|c|c|c|c|}
\hline TAV & Analyte & $\begin{array}{l}\leq 3.9 \mathrm{~cm} \\
(\mathrm{n}=38)\end{array}$ & $\begin{array}{c}4.0-5.9 \mathrm{~cm} \\
(\mathrm{n}=29)\end{array}$ & $\begin{array}{l}\geq 6.0 \mathrm{~cm} \\
(\mathrm{n}=17)\end{array}$ \\
\hline \multirow[t]{3}{*}{ Collagenases } & MMP-1 & 83 (103) & $51(57)^{*}$ & $65(52)$ \\
\hline & MMP-8 & $69(77)$ & $87(91)$ & $120(68)$ \\
\hline & MMP-13 & $50(133)$ & $117(345)^{*}$ & $30(111) \dagger$ \\
\hline \multirow[t]{2}{*}{ Gelatinases } & MMP-2 & $106(67)$ & $91(42)$ & $92(33)$ \\
\hline & MMP-9 & 45 (89) & 79 (107) & 56 (101) \\
\hline \multirow[t]{2}{*}{ Lysins } & MMP-3 & $67(81)$ & $38(24)^{*}$ & $55(58)$ \\
\hline & MMP-7 & $85(80)$ & $86(91)$ & $167(223)^{*}$ \\
\hline \multirow[t]{2}{*}{ Membrane type } & MMP-14 & 75 (114) & $95(64)$ & $80(106)$ \\
\hline & MMP-15 & $85(34)$ & $74(57)$ & $89(53)$ \\
\hline Elastase & MMP-12 & $74(67)$ & $67(62)$ & $70(73)$ \\
\hline \multirow[t]{4}{*}{ Inhibitors } & TIMP-1 & 72 (99) & $104(176)$ & $76(56)$ \\
\hline & TIMP-2 & $80(41)$ & $50(33)^{*}$ & $61(49)$ \\
\hline & TIMP-3 & $48(77)$ & $49(126)$ & 58 (127) \\
\hline & TIMP-4 & 79 (67) & $104(81)$ & $99(91)$ \\
\hline
\end{tabular}

$M M P$, Matrix metalloproteinase; TIMP, tissue inhibitors of metalloproteinase; TAV, tricuspid aortic valve. $* P 0.05$ vs $<3.9-\mathrm{cm}$ group. $\dagger P<.05$ vs 4.0-5.9-cm group. only when the entire cohort of samples was considered as 1 group. When aneurysm size was indexed by body surface area, a positive size correlation was found only for MMP-8 $(P=.04)$ in the BAV group. No significant relationships were observed between MMP levels and valve pathology.

\section{Discussion}

The congenitally bicuspid aortic valve is the most common congenital cardiovascular malformation, affecting $0.9 \%$ to $2 \%$ of the general population. ${ }^{11}$ Congenital BAVs are prone to development of both stenosis and regurgitation and are associated with aortic pathology such as coarctation and dissection, in addition to an increased incidence of ATAAs. ${ }^{11}$ Current hypotheses with regard to BAV-related ATAA formation include genetic abnormalities in the neural crest-origin cells in the ascending aorta and the flow hypothesis, in which turbulent blood flow across the BAV results in hemodynamic stress on the ascending aortic wall, especially the right anterolateral (outer) convexity. ${ }^{11,12}$ Although the histopathologic description of the manifestations of BAV with respect to ATAAs is improving, ${ }^{12}$ the underlying biochemical basis for these structural changes within the extracellular matrix remains poorly understood. It is now recognized that alterations in a specific extracellular matrix proteolytic cascade involving the MMPs and TIMPs occur in various cardiovascular disease states such as abdominal aortic aneurysms as well as ATAAs. ${ }^{5,6}$ However, a comparative, comprehensive quantitation of MMPs and TIMPs that occur within ATAAs in BAV with respect to TAV has not been performed. Accordingly, the present study measured representative MMPs from each of the major classes of this metalloprotease family, as well as all of the currently known TIMPs in a sample of ATAAs resected from patients with BAV

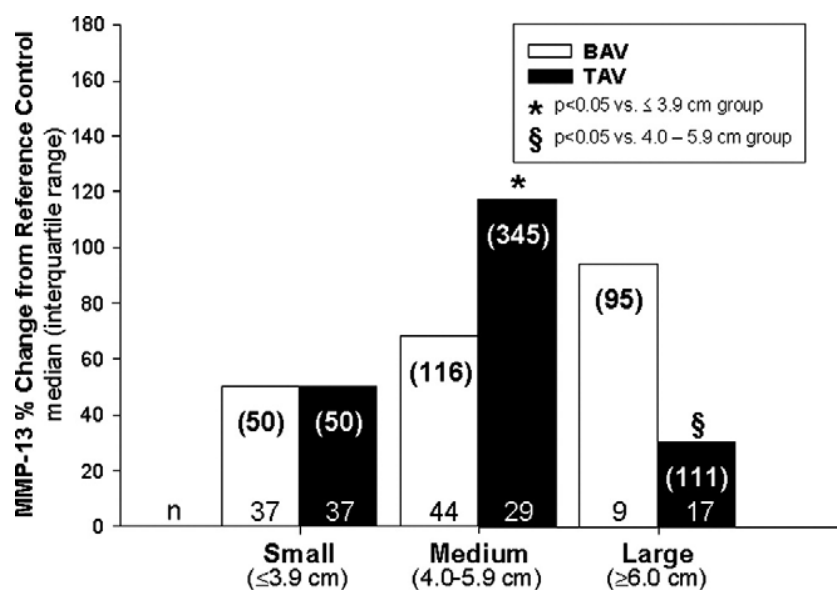

Figure 6. Comparison of MMP-13 levels for BAV and TAV aneurysms by aneurysm size. MMP-13 was increased in the TAV cohort for medium-sized aneurysms compared with the small aneurysm group. The large group showed a significant decrease in MMP-13 compared with the medium sized aneurysm group. $M M P$, Matrix metalloproteinase; BAV, bicuspid aortic valve; TAV, tricuspid aortic valve. 
and TAV. When compared with reference control patients, the unique findings from this study were that specific MMP and TIMP profiles emerged in the ATAAs from patients with BAV compared with the patients with TAV. For example, significantly increased MMP-2 and decreased membrane type-1 MMP (MMP14) occurred in the ATAAs from patients with patients with BAV. In contrast, the TAV aortic samples showed increased MMP-13 and decreased TIMP-2. These findings, with respect to processing of extracellular matrix constituents as well as other biologic signaling molecules, would suggest that a unique proteolytic process occurs in the development of ATAAs in patients with BAV, which may hold future diagnostic and prognostic implications.

Several studies to date have compared MMP and TIMP expression in BAV versus TAV aortic samples. ${ }^{1-4}$ Fedak and colleagues ${ }^{1}$ reported increased MMP-2 in BAV aortic samples compared with TAV samples with no changes in MMP-9 between the 2 groups. Boyum and colleagues ${ }^{2}$ demonstrated both increased MMP-2 and MMP-9 in BAV samples, with no differences in TIMP-1 and TIMP-2 relative to TAV aortic tissue. Koullias and associates ${ }^{3}$ examined valve tissue from patients with BAV and TAV, of which half of the population required a concomitant aortic procedure. The results showed increased MMP-2 and MMP-9 in the BAV tissue with nonsignificant trends toward increased TIMP-1 and TIMP-2 compared with the patients with TAV. Lemaire and coworkers ${ }^{4}$ compared ATAA tissue from patients with BAV and patients with TAV to control ascending aortic samples and found increased MMP-2 in the BAV samples compared with TAV and control and increased MMP-9 in the TAV samples compared with BAV and control, with no differences in TIMP-1 and TIMP-2. The results mentioned earlier raise several points. First, of the greater than 25 known MMPs and 4 TIMPs, ${ }^{13}$ the studies mentioned earlier provide a limited profile of MMP and TIMP types in BAV and TAV tissue. The present study contributes to the data described earlier by providing a more comprehensive survey of the known MMP classes in addition to all 4 TIMPs. Second, there is disparity with regard to the involvement of MMP-9 in the pathogenesis of these common forms of ATAA, in contrast to abdominal aortic aneurysms where MMP-9 is considered to be very important. ${ }^{5,6}$ The present study showed little change in MMP-9 in either BAV or TAV tissue. Third, each study discussed earlier showed an increase in MMP-2 in the BAV group, which is also supported by the findings of the present study. Finally, in contrast to the previous studies, the larger sample size of this study allowed comparison of numerous MMPs and TIMPs by increasing aneurysm size.

\section{MMPs}

For BAV samples, the significant findings of this study were increased MMP-2 and decreased MMP-14 overall, with these results seen particularly in the $4.0-$ to $5.9-\mathrm{cm}$ size range. MMP-2, or gelatinase $\mathrm{A}$, is synthesized by resident cells in the aortic wall such as fibroblasts, smooth muscle cells, and endothelial cells. ${ }^{13}$ It is not released by inflammatory cells such as neutrophils and macrophages, and recent work has demonstrated a reduced inflammatory infiltrate seen in BAV-associated ATAAs compared with TAV-associated ATAAs, consistent with these observations. ${ }^{4} \mathrm{MMP}-2$, in addition to its elastolytic properties, also cleaves numerous collagen subtypes (I-V, XI), laminin, and fibrillin-1. ${ }^{14,15}$ It has recently been shown that extracellular matrix remodeling and dilatation of the ascending aorta in patients with BAV occurs in an asymmetrical fashion, which is most pronounced in the right anterolateral aspect (convexity). ${ }^{12}$ The study by Della Corte and colleagues ${ }^{12}$ demonstrated a reduction in collagens I and III in addition to laminin in the outer convexity compared with the inner concavity of BAV-associated ATAAs, consistent with MMP-2 activity. Further, Fedak and associates ${ }^{1}$ showed decreased fibrillin-1 in BAV-associated ATAA tissue consistent with the finding of increased MMP-2 observed in this population. In addition, it is well described that MMP-2 is activated in response to turbulent flow and shear stresses such as those seen across a BAV and disproportionally in the outer curvature of the ascending aorta. ${ }^{16}$ However, the present study showed no statistical correlation between valve pathology and aneurysm formation in either valve group, suggesting that the turbulent flow relationship may be 1 of many factors acting in concert to form aneurysms.

With regard to MMP-2 activation, it is interesting that MMP-14 is decreased in the setting of an increase of MMP-2 in this population. One major function of MMP-14 is the activation of MMP-2 by a mechanism involving TIMP-2. ${ }^{17,18}$ Although not quantified, the immunoblotting results (Figure 1) showed an increase in the active form $(66 \mathrm{kd})$ of MMP-2 in BAV-associated ATAA tissue, suggesting increased MMP-2 activity. When taken together with the observed decrease in MMP-14, this suggests that an alternative mechanism of MMP-2 activation is operational, a finding that warrants further investigation.

For the TAV samples, perhaps the most significant finding is that of increased MMP-13 in the 4.0- to 5.9-cm size range, with a decrease in this species with larger aneurysm size. MMP-13, or collagenase-3, is a powerful proteinase produced by native cells in the aortic wall, and its expression is strictly controlled under normal physiologic conditions. It has a broad substrate specificity including many collagen types (I-IV, IX, X, XIV), tenascin C, fibronectin, aggrecan, versican, and osteonectin. ${ }^{18} \mathrm{In}$ addition, MMP-13 can inactivate chemokines, such as monocyte chemoattractant protein-3 and stromal cellderived factor-1. ${ }^{19}$ MMP-13 also appears to be involved in the activation of pro-transforming growth factor- $\beta 3$. MMP-13 expression is also detected in pathologic condi- 
tions that are characterized by the destruction of normal collagen-containing tissue architecture, including abdominal aortic aneurysms. ${ }^{20}$ The unique finding of increased MMP-13 in the TAV group in this study is consistent with extracellular matrix destruction and perhaps an active expansion phase in medium-sized ATAAs. As the aneurysm grows further, there may be a sufficient decline of resident cells (by senescence and apoptosis) to decrease the amount of MMP-13 produced, explaining the decline in MMP-13 demonstrated for larger aneurysm sizes. In addition, regression analysis showed a positive correlation between MMP-13 and age. Previous work has shown that tissue MMP levels are age sensitive, ${ }^{13,14}$ and the associative result shown in the present study suggests that MMP-13 may be even more important for aneurysm formation in older patients.

Another interesting finding with the TAV group is the demonstration of increased MMP-7 in large aneurysms. MMP-7, or matrilysin, is a small-molecular-weight MMP elaborated by many cell types, both constitutive and inflammatory. It serves numerous functions including extracellular matrix degradation (elastin, type IV collagen, fibronectin, vitronectin, aggregan, and proteoglycans), $\mathrm{MMP}(-1,-2$, -9) activation, and regulation of bioactive molecules controlling inflammation, cell proliferation, apoptosis, invasion, and angiogenesis. ${ }^{21}$ Of potential relevance to the present study is the ability of MMP-7 to cleave tumor necrosis factor (TNF)- $\alpha$ precursor to release soluble TNF- $\alpha$, which, through binding to TNF-receptor 1, may increase apoptosis. In abdominal aortic aneurysms, increased MMP-7 levels have been demonstrated only in the aneurysm wall and not luminal thrombus, suggesting that MMP-7 was elaborated more likely by resident cells within the aortic wall than by inflammatory cells. ${ }^{22,23}$ As such, MMP-7 may play an important late role on aneurysm progression though promotion of continued cellular attrition within the aortic wall.

In general, the derangements described earlier occurred for MMPs that are constitutively expressed by cells resident within the aortic wall (fibroblasts, smooth muscle cells). There appears, at least from this analysis, to be less of a proteolytic contribution from those MMPs elaborated from inflammatory cells (eg, MMP-8, MMP-9, MMP-12) for the ascending aorta than that seen in the abdominal aorta. ${ }^{5,24,25}$ It is possible that in response to pathologic stimuli, the resident cells undergo a pathologic transformation, resulting in increased MMP synthesis and release with increasing aneurysm size. Additional studies are required to address this hypothesis.

\section{TIMPs}

Primary endogenous control of MMP activity is maintained through 4 TIMPs. Each member of the TIMP family exhibits a distinct pattern of affinity for particular MMPs through a reversible, 1:1 interaction, ${ }^{7}$ and stoichiometry shifts between MMPs and TIMPs have been shown to favor abdominal aortic aneurysm formation. ${ }^{6,12}$ In addition, TIMPs have a number of other effects including activation of other MMPs, potentiation of growth factors, and instigation or suppression of apoptosis. ${ }^{7}$ In the present study, a complete survey of all 4 TIMPs was undertaken including TIMP-1, highly expressed in the aorta, ${ }^{6,12}$ and the cardiovascular system-specific TIMP-4. ${ }^{26}$ In the present study, TIMP-1 was decreased in the BAV group in the $\geq 6.0$-cm size range and TIMP-2 was decreased in the TAV group in the 4.0- to 5.9-cm size range. Decreased TIMPs in the setting of increased MMPs as shown earlier could represent a stoichiometry shift of proteolytic activity versus endogenous inhibition favoring extracellular matrix breakdown and ATAA growth.

\section{Clinical Significance}

The present study identified specific and different MMP and TIMP species produced in tissue of BAV- versus TAVassociated ATAAs. These species may provide important therapeutic targets in that tailored inhibitory strategies aimed at these MMPs may decrease or halt ATAA formation while minimizing the untoward side effect profiles typically associated with global MMP inhibition. Also, novel imaging or blood-testing techniques may be developed in which specific MMP imaging within the aorta may be used for diagnostic and prognostic assessment. For example, in the TAV group, imaging or plasma testing showing increased MMP-13 could indicate a medium-sized ATAA in a growth phase, and the later emergence of MMP-7 could then indicate a more mature, larger-sized ATAA with a different expansion rate.

Because this study was performed on resected aortic specimens at the time of surgery, inherent limitations exist. First, it is unclear whether the MMP and TIMP profiles measured are reflective of a specific stage in aneurysm progression or represent a summary of multiple phases. As stated earlier, further studies are required to develop other techniques of aneurysm characterization such as specific MMP plasma assays or imaging modalities to more carefully address this limitation. In addition, the potential for regional aortic heterogeneity implies that MMP and TIMP profiles seen in the ascending aorta may be different from the aortic arch or descending thoracic aorta and hence are not translatable. Also important is that caution must be exercised in extrapolating these MMP measurement results to true matrix-degrading activity and thus a more direct assessment of activity is required. By strict measurement criteria, some of the samples obtained were not from aortas that would truly be considered to be aneurysmal. However, assessment of these patients is also important because this represents the early stages of aneurysm formation where an 
early intervention may reduce, arrest, or reverse the dilatation process. Although different MMP cassettes were demonstrated for BAV- and TAV-associated ATAA tissue, a true cause-effect relationship between these changes and aneurysm expansion was not demonstrated and thus further studies are warranted. Comparison of aneurysmal aorta to adjacent nondilated aorta could be helpful in this regard. Finally, careful examination of the inner and outer curvature of the aneurysm wall is required to define regional MMP and TIMP profiles as they relate to disparities in extracellular matrix remodeling within the aneurysm.

Nevertheless, the results from the present study imply that a unique and differential extracellular matrix proteolytic signature exists in within the aorta of patients with BAV as opposed to patients with TAV with ascending aortic aneurysm disease and suggests that these disease processes do not follow a common etiology.

\section{References}

1. Fedak PWM, de Sa MPL, Verma S, Nili N, Kazeman P, Butany J, et al. Vascular matrix remodeling in patients with bicuspid aortic valve malformations: implications for aortic dilatation. J Thorac Cardiovasc Surg. 2003;126:797-806.

2. Boyum J, Fellinger EK, Schmoker JD, Tromblet L, McPartland K, Ittleman FP, et al. Matrix metalloproteinases activity in thoracic aortic aneurysms associated with bicuspid and tricuspid aortic valves. J Thorac Cardiovasc Surg. 2004;127:686-91.

3. Koullias GJ, Korkolis DP, Ravichandran P, Psyrri A, Hatzaras I, Elefteriades JA. Tissue microarray detection of matrix metalloproteinases, in diseased tricuspid and bicuspid aortic valves with or without pathology of the ascending aorta. Eur J Cardiothorac Surg. 2004;26: 1098-103.

4. LeMaire SA, Wang X, Wilks JA, Carter SA, Wen S, Won T, et al. Matrix metalloproteinases in ascending aortic aneurysms: bicuspid versus trileaflet aortic valves. J Surg Res. 2004;123:40-8.

5. Thompson RW, Holmes DR, Mertens RA, Liao S, Botney MD, Mecham RP, et al. Production and localization of 92-kilodalton gelatinase in abdominal aortic aneurysms. An elastolytic metalloproteinase expressed by aneurysm-infiltrating macrophages. J Clin Invest. 1995; 96:318-26.

6. Tamarina NA, McMillan WD, Shively VP, Pearce WH. Expression of matrix metalloproteinases and their inhibitors in aneurysms and normal aorta. Surgery. 1997;122:264-71.

7. Baker AH, Edwards DR, Murphy G. Metalloproteinase inhibitors: biological actions and therapeutic opportunities. J Cell Sci. 2002;115: 3719-27.

8. Spinale FG, Coker ML, Thomas CV, Walker JD, Mukherjee R, Hebbar L. Time dependent changes in matrix metalloproteinase activity and expression during the progression of congestive heart failure: relation to ventricular and myocyte function. Circ Res. 1998;82:482-95.

9. Wilson EM, Moainie SL, Baskin JM, Lowry AS, Deschamps AM, Mukherjee R, et al. Region- and type-specific induction of matrix metalloproteinases in post-myocardial infarction remodeling. Circulation. 2003;107:2857-63.

10. Davies RR, Gallo A, Coady MA, Tellides G, Botta DM, Burke B, et al. Novel measurement of relative aortic size predicts rupture of thoracic aortic aneurysms. Ann Thorac Surg. 2006;81:169-77.

11. Braverman AC, Guven H, Beardslee MA, Makan M, Kates AM, Moon MR. The bicuspid aortic valve. Curr Probl Cardiol. 2005;30:470-522.

12. Della Corte A, DeSanto LS, Montagnani S, Quarto C, Romano G, Amarelli C, et al. Spatial patterns of matrix protein expression in dilated ascending aorta with aortic regurgitation: congenital bicuspid valve versus Marfan's syndrome. J Heart Valve Dis. 2006;15:20-7.
13. Thompson RM. Abdominal aortic aneurysms: basic mechanisms and clinical implications. Curr Probl Surg. 2002;39:110-230.

14. Nagase H, Visse R, Murphy G. Structure and function of matrix metalloproteinases and TIMPs. Cardiovasc Res. 2006;69:562-73.

15. Ashworth JL, Murphy G, Rock MJ, Sherrat MJ, Shapiro SD, Shutleworth CA, et al. Fibrillin degradation by matrix metalloproteinases: implications for connective tissue remodeling. Biochem J. 1999;340: 171-81.

16. Gambillara V, Montorzi G, Haziza-Pigeon C, Stergiopulos N, Silacci P. Arterial wall response to ex vivo exposure to oscillatory shear stress. J Vasc Res. 2005;42:535-44.

17. Strongin AY, Collier I, Bannikov G, Marmer BL, Grant GA, Goldberg GI. Mechanism of cell surface activation of 72-kDa type IV collagenase. Isolation of the activated form of the membrane metalloprotease. J Biol Chem. 1995;270:5331-8.

18. Wang Z, Juttermann R, Soloway PD. TIMP-2 is required for efficient activation of proMMP-2 in vivo. J Biol Chem. 2000;275:26411-5.

19. Ala-aho R, Kahari V-M. Collagenases in cancer. Biochimie. 2005;87: 273-86.

20. Mao D, Lee JK, VanVickle SJ, Thompson RW. Expression of collagenase-3 (MMP-13) in human abdominal aortic aneurysms and vascular smooth muscle cells in culture. Biochem Biophys Res Commun. 1999;261:904-10.

21. Ii M, Yamamoto H, Adachi Y, Maruyama Y, Shinomura Y. Role of matrix metalloproteinase-7 (matrilysin) in human cancer invasion, apoptosis, growth and angiogenesis. Exp Biol Med. 2006;231:20-7.

22. Fontaine V, Jacob MP, Houard X, Rossignol P, Plissonnier D, AngelsCano E, et al. Involvement of the mural thrombus as a site of protease release and activation in human aortic aneurysms. Am J Pathol. 2002; 161:1701-10.

23. Kazi M, Zhu C, Roy J, Paulsson-Berne G, Hamsten A, Swedenborg J, et al. Difference in matrix-degrading protease expression and activity between thrombus-free and thrombus-covered wall of abdominal aortic aneurysm. Arterioscler Thromb Vasc Biol. 2005;25:1341-6.

24. Longo GM, Xiong W, Greiner TC, Zhao Y, Fiotti N, Baxter BT. Matrix metalloproteinases 2 and 9 work in concert to produce aortic aneurysms. J Clin Invest. 2002;110:625-32.

25. Longo GM, Buda SJ, Fiotta N, Xiong W, Greiner TC, Shapiro S, et al. MMP-12 has a role in abdominal aortic aneurysms in mice. Surgery. 2005; $137: 457-62$

26. Stroud RE, Deschamps AM, Lowry AS, Hardin AE, Mukherjee R, Lindsey ML, et al. Plasma monitoring of the myocardial specific tissue inhibitor of metalloproteinase- 4 after alcohol septal ablation in hypertrophic obstructive cardiomyopathy. J Card Fail. 2005;11:124-30.

\section{Appendix}

\begin{tabular}{llcl}
\hline Species & Catalog \# & Dilution & \multicolumn{1}{c}{ Company* } \\
\hline MMP-1 & IM35L & $1: 2500$ & Oncogene \\
MMP-3 & IM36L & $1: 5000$ & Oncogene \\
MMP-7 & PC492 & $1: 2500$ & Oncogene \\
MMP-8 & $358-100$ & $1: 2500$ & Biovision \\
MMP-12 & RP1-MMP12 & $1: 2500$ & Triple Point \\
MMP-13 & RP3-MMP13 & $1: 2500$ & Triple Point \\
MMP-14 & AB815 & $1: 5000$ & Chemicon \\
MMP-15 & RP2-MMP-15 & $1: 2500$ & Triple Point \\
TIMP-1 & OPA1-08512 & $1: 2500$ & Affinity \\
TIMP-2 & IM11L & $1: 2500$ & Oncogene \\
TIMP-3 & AB802 & $1: 1000$ & Chemicon \\
TIMP-4 & AB816 & $1: 2500$ & Chemicon \\
\hline MMP, Matrix & metalloproteinase; & TIMP, tissue & inhibitors of \\
metalloproteinase. *Company information: Oncogene, Boston, Mass; Bio- \\
vision, Mountain View, Calif; Triple Point Biologics, Forest Green, Ore; \\
Chemicon, Temecula, Calif; Affinity Bioreagents, Golden, Colo.
\end{tabular}




\section{Discussion}

Dr John A. Kern (Charlottesville, $\mathrm{Va}$ ). John, that was a very nice study that gives us more insight into the molecular biology of ascending aortic aneurysm pathology. As you detailed in your manuscript, but not so much here because of time constraints, your results really parallel those of others. However, your study goes a bit further in showing us various activity profiles even among aneurysms of different sizes, which is quite interesting.

The main questions I have, to bring this into some clinical appreciation, is what does this study really do in furthering our understanding of the natural history of ascending aortic aneurysms, and will findings such as these ultimately change the way we approach aneurysms, particularly with respect to timing of repair?

I also have a few nuts-and-bolts questions. The patients in the control group were quite a bit younger than those in the other groups, and it makes us wonder about the biology of MMPs in general, so could you just talk a bit about that. Also, your findings may have a genetic basis. Did you see any differences in profiles with respect to gender?

In addition, there is potentially a lot of differing medical therapy in these patients, so I' $m$ wondering, as the patients weren't controlled for things like antihypertensive therapy and antilipid therapy, could those factors have had an impact on your results?

And last, obviously what we're all interested in is trying to figure out which patients are getting ready to have an aortic catastrophe. So is there any way we can use these results-in other words, are any of these molecules considered such acute phasetype reactants, that we could somehow formulate an assay, whether it be a serum assay or an imaging test, that we could use to find those patients who are getting ready to become unstable? And then, if so, could we use these concepts to formulate some form of therapy, whether it be through conventional techniques or endovascular techniques?

Dr John S. Ikonomidis (Charleston, SC). Thank you, Dr Kern, for raising those very pertinent issues. In answer to your overall question, I think that we continue to further our knowledge about biology of ATAA disease; specifically, we are starting to tease out molecular pathways and effectors not just for 1 aneurysm type but now among various pathologic types.

I think that the present study adds to previous studies in the sense that we have examined a broader range of MMPs and TIMPs and provided, perhaps, a larger profile. And in addition, we have had somewhat of a larger sample size, which allowed some correlations to be made to aneurysm size, although I should preface that by saying that Dr Fedak has already demonstrated at the $P=$ .05 level an association between MMP-2 and bicuspid ascending aortic aneurysms.

As far as age is concerned, there is no doubt that age within the cardiovascular system has an effect on MMP biology. And this is a confounding factor in this study that would be difficult to remove. This point is offset, to some extent, by the fact that we were able to examine specific aneurysm types by size within each group.

MMPs, in general, are not considered acute-phase reactants. They are extremely tightly regulated molecules that are active in both healthy and diseased states. In our animal model, we saw upregulation of MMP-2 and MMP-9 within approximately 72 hours of an aneurysm stimulus.

As far as gender is concerned, we did analyze these results by gender and found no statistical differences.

There are certain medical therapies that would modify MMP activity. For example, treatment of these patients with betablockers and the resultant decrease in shear stresses and $\mathrm{dP} / \mathrm{dt}$ would decrease certain MMPs that are stress dependent, particularly MMP-2. This could have a significant impact in therapy for these aneurysms, much the same as there is a salutary effect in Marfan's syndrome.

None of these aneurysms came from patients who had an impending catastrophe, so I'm not able to provide profiles to you about those particular patients; however, development of specific plasma or radiologic imaging strategies may allow us to characterize the MMP profiles over time such that with further study, we may be able to identify a specific MMP and TIMP cassette that may indicate an impending rupture or a change in the character of the aneurysm. And to that end, enzyme-linked immunosorbent assay tests are already being developed for following MMPs and TIMPs such as MMP-9, MMP-2, and TIMP-1 post-myocardial infarction, such that these may also be an option for following patients with thoracic aortic aneurysm disease.

Furthermore, radiolabeled short-acting inhibitors, either global or specific, bind within the cardiovascular system and can be imaged. We may be able to use these for serial imaging to follow either global MMP activity or specific MMPs as we tease out the different MMP profiles within the different aneurysm groups.

And finally, how can we make this work for us? These data may help us with diagnosis and prognostication. Also, novel therapeutic strategies could include development of specific MMP inhibitors that target $1 \mathrm{MMP}$ or a specific subgroup of MMPs out of the 26 known species, thus reducing the side-effect profiles that are classically seen with global MMP inhibition. Obviously, gene therapy is also a possibility.

Endovascular stent grafting is a very hot therapeutic modality right now; but a potential problem with this treatment modality is that the stent-grafted aorta likely continues to remodel. Thus, over time, the stent graft may fail through continued remodeling of the aneurysm and aorta both proximal and distal to the stent-graft. It therefore may be valuable to place MMP inhibitors within the fabric of the aneurysm to arrest remodeling and thus increase the longevity of the stent-graft repair.

Dr Doosang Kim (Seoul, Korea). My question is about the location of the sample site. Where is the most common location of the sample site?

Dr Ikonomidis. The sample site was taken at the point where the aneurysm was largest by inspection at the time of resection. 